\title{
Commentary: High-intensity Intermittent Training vs. Moderate-intensity Intermittent Training: Is It a Matter of Intensity or Intermittent Efforts?
}

\author{
Paulo Gentil ${ }^{1 *}$ and Fabrício B. Del Vecchio ${ }^{2}$ \\ ${ }^{1}$ Faculdade de Educação Física e Dança, Universidade Federal de Goiás, Goiânia, Brazil, ${ }^{2}$ Escola Superior de Educação \\ Física, Universidade Federal de Pelotas, Pelotas, Brazil
}

Keywords: high intensity interval exercise, high intensity intermittent training, physical fitness, high intensity exercise, cardiorespiratory fitness

\section{A commentary on}

High-intensity intermittent training versus moderate-intensity intermittent training: is it a matter of intensity or intermittent efforts?

by Jimenéz-Pavón, D., and Lavie, C. J. (2017). Br. J. Sports Med. doi: 10.1136/bjsports-2016-097015. [Epub ahead of print]

OPEN ACCESS

Edited by:

Billy Sperlich,

University of Würzburg, Germany

Reviewed by:

Thimo Wiewelhove,

Ruhr University Bochum, Germany

*Correspondence:

Paulo Gentil

paulogentil@hotmail.com

Specialty section:

This article was submitted to

Exercise Physiology,

a section of the journal

Frontiers in Physiology

Received: 12 April 2017 Accepted: 18 May 2017

Published: 30 May 2017

Citation:

Gentil P and Del Vecchio FB (2017)

Commentary: High-intensity

Intermittent Training vs.

Moderate-intensity Intermittent

Training: Is It a Matter of Intensity or

Intermittent Efforts?

Front. Physiol. 8:370

doi: 10.3389/fphys.2017.00370
We read with great interest the article by Jimenéz-Pavón and Lavie (2017). While we agree with the value of prescribing and studying moderate-intensity interval training (MIIT), there are important aspects that need to be clarified. The authors raise the point that "intensity" is not the only difference between high-intensity interval training (HIIT) and aerobic continuous training (ACT). The authors cite three studies to support the notion that the intermittent nature of the exercise and not the intensity may be responsible for the results. However, the studies cited do not seem to support the points raised. Rakobowchuk et al. (2012) trained two groups at the same intensity ( $120 \%$ of the peak work rate obtained in a ramp-incremental test), but with different working parameters (repeated intervals of 10:20 s vs. intervals of 30:60 s); therefore, this cannot be considered a comparison between HIIT and MIIT. In the study conducted by Alkahtani et al. (2013), the protocols differed in intensity but also in interval duration ( $5 \mathrm{~min}$ vs. $30 \mathrm{~s}$ ). Therefore, one cannot use the study to make inferences about training intensity as the other parameters were not equal. Moreover, in the study conducted by Alkahtani et al. (2013), the group that exercised at higher intensity performed $30 \mathrm{~s}$ at $90 \%$ of the intensity equivalent to VO2peak, interspersed with $30 \mathrm{~s}$ of passive rest. Although this intensity was higher than that performed by the other group (20\% above $45 \%$ VO2peak), it is still lower than reported in previous studies using the same interval duration (Billat et al., 1999, 2000a,b; Billat, 2001a,b; Racil et al., 2013, 2016).

The only study that has really compared different intensities is Racil et al. (2013). The authors equated the number of bouts, rest intervals, etc. In this study, the decreases in waist circumference, triglyceride and total cholesterol were significant only in the HIIT group. In addition, increases in maximal aerobic speed and decreases in percentage of body fat, low-density lipoprotein cholesterol (LDL-C), and insulin were higher in the HIIT group than in the MIIT group. Although the increases in VO2peak were significant for both groups (7.7\% for HIIT and 5.2 for MIIT), only the HIIT group showed a significant increase compared to the control group. Therefore, the only study that made comparisons with different intensities while keeping the other parameters constant clearly favored HIIT exercises. While this may not answer the question of whether intensity or the intermittent 
nature of interval training is the most important parameter, it suggests that HIIT promotes better results than MIIT.

We must recall, however, that defining HIIT intensity is not a matter of "the more the better." The efficiency of HIIT seems to be a matter of choosing the adequate intensity. In this regard, Raleigh et al. (2016) investigated the effects of HIIT intensity on training-induced adaptations in VO2peak and VO2 kinetics. The authors compared the effects of HIIT ( 1 min of effort per $1 \mathrm{~min}$ of rest) targeting 80,115 , or $150 \%$ of the intensity equivalent to $\mathrm{VO} 2 \mathrm{max}$ while matching total work performed. According to the results, increases in VO2peak were greater in the group that trained at $115 \%$ than in the group that trained at $80 \%$. No differences were observed between the groups that trained at 150 and $80 \%$ as well as between the groups that trained at 150 and $115 \%$ of iVO2max. The greatest proportion of non-responders was observed in the group that trained at lower intensity and the greatest proportion of responders was found in the group that trained at $115 \%$. Therefore, one should not advocate for or against high intensity, but rather for adequate intensity.

Jimenéz-Pavón and Lavie (2017) suggested that high-intensity exercise can sometimes deter physically inactive and unfit people; however, this has not been found in previous studies. Indeed, Guiraud et al. (2011) reported that patients with chronic heart disease preferred HIIT to ACT. Jung et al. (2014) reported that adults with prediabetes can adhere to HIIT at a level that is

\section{REFERENCES}

Alkahtani, S. A., King, N. A., Hills, A. P., and Byrne, N. M. (2013). Effect of interval training intensity on fat oxidation, blood lactate and the rate of perceived exertion in obese men. Springerplus 2:e532. doi: 10.1186/2193-1801-2-532

Billat, L. V. (2001a). Interval training for performance: a scientific and empirical practice. Special recommendations for middle- and long-distance running. Part I: aerobic interval training. Sports Med. 31, 13-31.

Billat, L. V. (2001b). Interval training for performance: a scientific and empirical practice. Special recommendations for middle- and long-distance running. Part II: anaerobic interval training. Sports Med. 31, 75-90.

Billat, V. L., Bocquet, V., Slawinski, J., Laffite, L., Demarle, A., Chassaing, P., et al. (2000a). Effect of a prior intermittent run at vVO2max on oxygen kinetics during an all-out severe run in humans. J. Sports Med. Phys. Fitness 40, 185-194.

Billat, V. L., Flechet, B., Petit, B., Muriaux, G., and Koralsztein, J. P. (1999). Interval training at VO2max: effects on aerobic performance and overtraining markers. Med. Sci. Sports Exerc. 31, 156-163.

Billat, V. L., Slawinski, J., Bocquet, V., Demarle, A., Lafitte, L., Chassaing, P., et al. (2000b). Intermittent runs at the velocity associated with maximal oxygen uptake enables subjects to remain at maximal oxygen uptake for a longer time than intense but submaximal runs. Eur. J. Appl. Physiol. 81, 188-196. doi: $10.1007 / \mathrm{s} 004210050029$

Guiraud, T., Nigam, A., Juneau, M., Meyer, P., Gayda, M., and Bosquet, L. (2011). Acute responses to high-intensity intermittent exercise in chd patients. Med. Sci. Sports Exerc. 43, 211-217. doi: 10.1249/MSS.0b013e3181ebc5de

Heisz, J. J., Tejada, M. G., Paolucci, E. M., and Muir, C. (2016). Enjoyment for high-intensity interval exercise increases during the first six weeks of training: implications for promoting exercise adherence in sedentary adults. PLOS ONE 11:e0168534. doi: 10.1371/journal.pone.0168534

Jimenéz-Pavón, D., and Lavie, C. J. (2017). High-intensity intermittent training versus moderate-intensity intermittent training: is it a matter of intensity or intermittent efforts? Br. J. Sports Med. doi: 10.1136/bjsports-2016-097015. [Epub ahead of print].

Jung, M. E., Bourne, J. E., and Little, J. P. (2014). Where does HIT fit? An examination of the affective response to high-intensity intervals in comparison to continuous moderate- and continuous vigorous-intensity greater than ACT. Furthermore, Jung et al. (2015) compared HIIT ( $1 \mathrm{~min} \sim 100 \% \mathrm{~W}$ peak and $1 \mathrm{~min} \sim 20 \% \mathrm{~W}$ peak for $20 \mathrm{~min}$ ), ACT at moderate intensity ( $\sim 40 \% \mathrm{~W}$ peak for $40 \mathrm{~min})$ and ACT at high intensity ( $\sim 80 \% \mathrm{~W}$ peak for $20 \mathrm{~min}$ ). According to the results, participants reported greater enjoyment related to HIIT compared to the other protocols and $62 \%$ of the participants reported a preference for engaging in HIIT.

Although we agree that enjoyment ratings might be reduced when HIIT is strenuous, chronic training may lead to increased enjoyment due to an increase in achievement. In this regard, Heisz et al. (2016) randomly assigned sedentary young adults to HIIT ( 1 min $90-95 \%$ peak HR followed by $1 \mathrm{~min}$ at $30 \%$ PPO for a total of $20 \mathrm{~min}$ ) or ACT (27.5 min $70-75 \%$ peak HR) for 6 weeks. Enjoyment of HIIT increased with training, whereas enjoyment of ACT remained constant but lower.

While we agree that MIIT might be an interesting strategy at some points and that more studies regarding the topic are needed, the references presented and the limitations raised by the authors do not seem to support the points raised.

\section{AUTHOR CONTRIBUTIONS}

PG and FD conceived, drafted, and revised the manuscript. All authors read and approved the final manuscript.

exercise in the exercise intensity-affect continuum. PLOS ONE 9:e114541. doi: 10.1371/journal.pone.0114541

Jung, M. E., Bourne, J. E., Beauchamp, M. R., Robinson, E., and Little, J. P. (2015). High-intensity interval training as an efficacious alternative to moderateintensity continuous training for adults with prediabetes. J. Diabetes Res. 2015:e191595. doi: 10.1155/2015/191595

Racil, G., Ben Ounis, O., Hammouda, O., Kallel, A., Zouhal, H., Chamari, K., et al. (2013). Effects of high vs. moderate exercise intensity during interval training on lipids and adiponectin levels in obese young females. Eur. J. Appl. Physiol. 113, 2531-2540. doi: 10.1007/s00421-013-2689-5

Racil, G., Zouhal, H., Elmontassar, W., Ben Abderrahmane, A., De Sousa, M. V., Chamari, K., et al. (2016). Plyometric exercise combined with high-intensity interval training improves metabolic abnormalities in young obese females more so than interval training alone. Appl. Physiol. Nutr. Metab. 41, 103-109. doi: 10.1139/apnm-2015-0384

Rakobowchuk, M., Harris, E., Taylor, A., Baliga, V., Cubbon, R. M., Rossiter, H. B., et al. (2012). Heavy and moderate interval exercise training alters low-flow-mediated constriction but does not increase circulating progenitor cells in healthy humans. Exp. Physiol. 97, 375-385. doi: 10.1113/expphysiol.2011.062836

Raleigh, J. P., Giles, M. D., Scribbans, T. D., Edgett, B. A., Sawula, L. J., Bonafiglia, J. T., et al. (2016). The impact of work-matched interval training on VO2peak and VO2 kinetics: diminishing returns with increasing intensity. Appl. Physiol. Nutr. Metab. 41, 706-713. doi: 10.1139/apnm2015-0614

Conflict of Interest Statement: The authors declare that the research was conducted in the absence of any commercial or financial relationships that could be construed as a potential conflict of interest.

Copyright (C) 2017 Gentil and Del Vecchio. This is an open-access article distributed under the terms of the Creative Commons Attribution License (CC BY). The use, distribution or reproduction in other forums is permitted, provided the original author(s) or licensor are credited and that the original publication in this journal is cited, in accordance with accepted academic practice. No use, distribution or reproduction is permitted which does not comply with these terms. 